\section{Zukunft durch \\ ökologische Dienstleistungen?}

\section{Zusammen mit dem Umzug des Regierungssitzes von Bonn nach Berlin werden auch Teile des dienstleistungsorientierten Umfeldes ihren Standort verlagern. Diese Entwicklung ist mit erheblichen wirtschaftlichen und sozialen Risiken verbunden. Das Institut für ökologische Wirtschaftsforschung untersuchte deshalb, welche Rolle ökologische Dienstleistungen zur Abmilderung dieses Prozesses spielen können.}

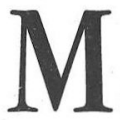

Von Rainer Lucas und Bettina Knothe Dienstleistungsstandort Bonn (Stadt Bonn, Rhein-Siegkreis, Kreis Ahrweiler) ein Kernbereich der wirtschaftlichen Aktivitäten verloren. Die daraus resultierenden Konsequenzen für die regionale Wertschöpfung und die Arbeitsplätze sind derzeit noch nicht abschätzbar. Doch die hiermit verbundenen wirtschaftlichen und sozialen Risiken sind in der Region erkannt und Maßnahmen für eine strategische Neuorientierung des Standortes eingeleitet. Die von der ÖTV-Bonn angeregte Vorstudie des Instituts für ökologische Wirtschaftsforschung (IÖW) konzentriert sich auf zwei Fragestellungen (1):

- Inwieweit ist die Region Bonn in der Lage, ökologische Standortfaktoren auszubauen und durch ein ökologisches Standortprofil Wettbewerbsvorteile zu erzielen?

- Welche Wachtumspotentiale existieren unter Berücksichtigung der allgemeinen Marktentwicklung und der spezifischen Fähigkeiten regionaler Anbieter im Bereich ökologische Dienstleistungen?

\section{Thematische}

\section{Rahmenbedingungen}

Unter ökologischen Dienstleistungen verstehen wir Leistungsangebote von Unternehmen und öffentlichen Einrichtungen, die direkt und indirekt dazu beitragen, Produktionsprozesse, Produkte und Dienstleistungen umweltfreundlicher zu gestalten. Die Umweltorientierung ist hierbei häufig mit anderen Problemstellungen verbunden. Integrationsfähigkeit und Kooperationsfähigkeit sind daher wichtige Voraussetzungen für die Erschließung der ökologischen Marktsegmente.
Die Bedeutung ökologischer Standortfaktoren insbesondere für Dienstleistungsunternehmen haben wir mit Bezug auf verschiedene Arbeiten und Studien zur tertiären Entwicklung der Branchen und zur Bedeutung umweltorientierter Standortfaktoren konzeptionell dargestellt. Die positive Dynamik, die aus einer Verbindung zwischen Standortattraktivität und der Ökologi-

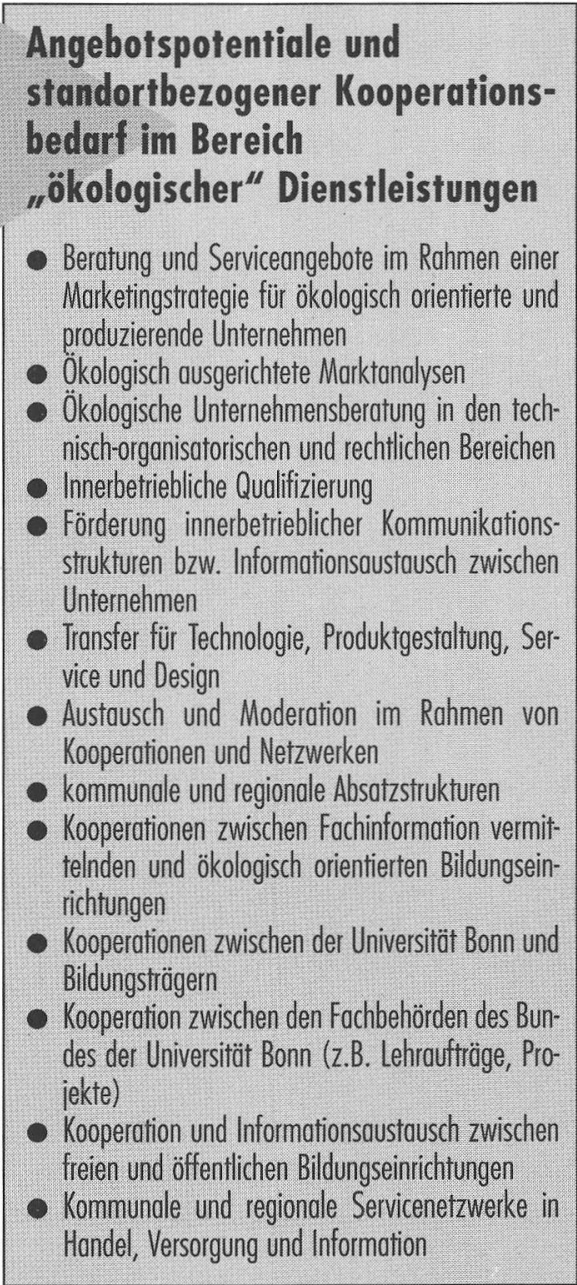

sierung des städtischen Wirtschaftens und Lebens entstehen kann, wurde herausgearbeitet. Ökologische Stadtentwicklungspolitik ist insbesondere auf die Herausbildung bzw. Erweiterung sogenannter weicher Standortfaktoren (Umweltqualität, Kultur und Freizeitangebote, Aus- und Weiterbildungsmöglichkeiten) angewiesen. Institutionelle Voraussetzungen hierfür sind eine stärkere Abstimmung der Ressorts Wirtschaftsförderung und Umwelt in der öffentlichen Verwaltung, eine bessere Nutzung der ökologischen Wissenspotentiale der wissenschaftlichen Einrichtungen sowie eine Ökologisierung des Dienstleistungsangebots seitens privater Unternehmen.

\section{Einschätzungen und Handlungspotentiale}

Zur Abschätzung der spezifischen Wachtumspotentiale in der Region Bonn wurde eine Expertenbefragung von regionalen Akteuren aus Wirtschaft, Wissenschaft, Verwaltung und Verbänden durchgefïhrt. Näher bestimmt wurden die Entwicklungspotentiale in den Handlungsfeldern Bildung/Weiterbildung, Beratungsleistungen für Unternehmen, konsumorientierte Beratungsleistungen für private Haushalte und logistische Dienstleistungen im Bereich Wirtschaftsverkehr. Die von uns befragten Experten hielten es zur Verbesserung der Wettbewerbsfähigkeit für angebracht, sich intensiver mit ökologischen Marktsegmenten zu befassen. Ökologische Produkte und Dienstleistungen, z.B. in der Produktlinie Ernährung oder im Bausektor, gewinnen in gesättigten, nachfragebestimmten Märkten zunehmend an Bedeutung. Für die Unternehmen im Raum Bonn bestehe daher im Verbund mit der Technologieförderung die Aufgabe, ökologische Problem- und Produktlösungen schneller und besser als potentielle Mitkonkurrenten zur Marktreife zu fuihren und konzeptionell umzusetzen (z.B. durch Demonstrationsvorhaben).

Für den Transfer bzw. den Austausch von wissenschaftlichem Know-How wurden von den Befragten folgende Einrichtungen präferiert:

- Forschungseinrichtungen der Region, z.B. Universität Bonn mit seinen Forschungsprojekten im vegetations- und bodenkundlichen sowie agrarwirtschaftlichen Bereich und dem Institut für Agrarpolitik, Marktforschung und Wirtschaftssoziologie;

- Bundesforschungsanstalt für Landes- und Raumentwicklung, Bundesamt für Naturschutz; 
Verwaltungsakademien; zentrale Einrichtungen der Umweltverbände.

Allgemein wurde die regionsinterne Kommunikation über ökologische Dienstleistungspotentiale in der Region Bonn als ausbaufähig eingestuft. Speziell bestand ein großes Interesse an einem Austausch über Zukunftstrends und Zukunftsmärkte. Ökologische Dienstleistungen decken ein vielfältiges Angebotsspektrum ab, welches teilweise auch schon erschlossen wurde. Die Potentiale dieses Bereiches ließen sich noch vergrößern, wenn systematischer als bisher die spezifischen Kooperationschancen zwischen der Privatwirtschaft und öffentlichen Einrichtungen genutzt würden.

Die ökologischen Wissenspotentiale der Region wurden als insgesamt gut eingeschätzt, allerdings sei es häufig schwierig, diese unmittelbar für die Praxis nutzbar zu machen und zusam- menzuführen. Folgende von den Interviewpartnern geäußerten Engpässe sollten abgebaut werden:

- Schaffung eines Problembewußtseins bei regionalen Dienstleistern und Produzenten,

- stärkere Anwendungsorientierung der Wissenschaft,

- Verbesserung des Wissenstransfers in die Praxis,

- Abbau der Berïhrungsängste zwischen ökologischen Dienstleistern und der traditionellen gewerblichen Wirtschaft und

- Verbesserung der Abstimmung zwischen den Akteuren zur Vermeidung parallel laufender Strategien.

\section{Literatur:}

(1) Vgl. Lucas, R./Knothe, B.: Zukunft des Dienstleistungszentrums Bonn - Ökologische Standortfaktoren und Entwicklungspotentiale ökologischer Dienstleistungen, IÖWSchriftenreihe 91/95, Berlin/Wuppertal 1995.

\section{Gründung einer Vereinigung für Ökologische Ökonomie im deutschsprachigen Raum}

\section{Call for members}

\section{Am 26. und 27. April 1996 soll in Heidelberg die deutschsprachige Sektion der International Society for Ecological Economics ins Leben gerufen werden.}

$\mathrm{W}$ Von Christiane Busch-Lüty Je Jahre der aktiven Mitwirkung auch auf Vorstandsebene, im altehrwürdigen „Verein für Socialpolitik“, der dominierenden, traditionsreichen, wissenschaftlichen Vereinigung der deutschsprachigen ökonomen, zurückblicken kann, der hat sich auch höchst persönlich davon überzeugen lassen müssen, daß der Ansatz einer dezidiert transdisziplinär ansetzenden und ganzheitlich ausgerichteten ökologischen Ökonomie dort auf absehbare Zeit keinerlei Chance fuir eine wissenschaftliche Beheimatung hat und haben wird.

\section{Transdisziplinäres Netzwerk}

So gibt es bisher keinen institutionell abgesicherten regelmäßigen Gedankenaustausch und erst recht keinerlei abgestimmte wissenschaftsund hochschulpolitische Aktionsmöglichkeiten der deutschsprachigen Ökologischen Ökonomen. Diese haben sich in den letzten Jahren zunehmend jeweils einzeln der stark expandierenden International Society for Ecological Eco- nomics, ISEE, angeschlossen. Während andere „Regional Chapters“ in aller Welt längst als Netzwerke ihr funktionales Eigenleben im Sinne der „Sustainability“ in der wissenschaftlichen wie der politischen Landschaft in Nord und Süd entfaltet haben, ist das Potential an ökologischen Ökonomen im deutschsprachigen Raum mit insgesamt 93 Mitgliedern (laut Membership Directority 1995 der ISEE: 49 in Deutschland, 14 in Österreich, 30 in der Schweiz) noch auffallend dünn und daher auch wenig wirksam für die überfälligen Veränderungsprozesse der wissenschaftlichen Ökonomie.

Angesichts des in den nächsten fünf bis zehn Jahren anstehenden Generationenwechsels auf den ökonomischen Lehrstühlen droht aber für weitere Jahrzehnte eine einseitige Weichenstellung in Richtung der traditionellen neoklassisch orientierten additiven Umwelt- und Ressourcenökonomie, wenn sich nicht die bisher wenigen Vertreter einer Ökologischen Ökonomie in Verbindung mit der starken Bewegung der ökologisch orientierten ökonomischen Studentengruppen sich stärker zusammen- schließen und strategisch handeln, um die Chance einer Veränderung der hochschulpolitischen Landschaft in Lehre und Forschung in ihrem Sinne zu nutzen.

\section{Konstituierung und Programm}

Deshalb hat eine kleine, interdisziplinär zusammengesetzte Wissenschaftlergruppe die Initiative für den Aufbau einer ,,Vereinigung für Ökologische Ökonomie" ergriffen, die zugleich als „Regional Chapter" unter dem Dach der ISEE angesiedelt und damit auch international optimal ausgestaltet werden soll. Dies gilt erst recht, nachdem für Ende Mai '96 in Paris die Konstituierung einer „European Branch" der ISEE vorgesehen ist.

Dank einer Starthilfe durch die Kapp-Stiftung kann nunmehr die Organisation und das Gründungstreffen einer solchen dezidiert interdisziplinär und intergenerational ausgerichteten Vereinigung für Ökologische Ökonomie konkret in die Wege geleitet werden: am 26. - 27. April 96 in der Forschungsstätte der Evangelischen Studiengemeinschaft (FEST) auf dem Heidelberger Schloßberg unter der - durchaus programmatischen - Schirm-Frauschaft von Heidelbergs Oberbürgermeisterin Beate Weber, der als Kommunal- wie Europapolitikerin bewährten „Praktikerin" in Sachen Ökologische Ökonomie.

Dieses Treffen soll neben der rechtlichen und organisatorischen Konstituierung vor allem der eigenen programmatischen Identitätsbestimmung dienen, aber auch Handlungsfelder konkretisieren (beispielsweise im Hinblick auf die im Informationsdienst 5-6/95 zum Themenschwerpunkt Ecological Economics von Jürgen Meyerhoff zu Recht beklagte „Leerstellé“ in der Lehrbuchlandschaft des deutschsprachigen Raums) und sich gegenüber der wissenschaftlichen Gemeinschaft und der Öffentlichkeit manifestieren.

\section{Call for members}

Interessentinnen werden gebeten, der unterzeichnenden Initiatorin bis spätestens zum 15. März eine Mitteilung zukommen zu lassen, wenn er/sie an einer Mirgliedschaft in der "Vereinigung für Ökologische Ökonomie" interessiert ist, und auch an dem Gründungstreffen in Heidelberg am 26./27.4.96 teilnehmen will (das aus Raumgründen auf ca. $40-50$ Personen beschränkt sein muß, daher ist eine möglichst frühzeitige Anmeldung wichrig)

Kontakt: Prof. Dr. Chr. Busch-Lüty, Posistr. 15, 82067 Ebenhausen//sar, Tel. (08178) 7688; Fax 7689 
(c) 20I0 Authors; licensee IÖW and oekom verlag. This is an article distributed under the terms of the Creative Commons Attribution Non-Commercial No Derivates License (http://creativecommons.org/licenses/by-nc-nd/3.o/), which permits unrestricted use, distribution, and reproduction in any medium, provided the original work is properly cited. 